Poznańskie Studia Teologiczne 28(2014), s. 47-65.

doi: $10.14746 /$ pst.2014.28.4

Stefano Tarocchi

Facoltà Teologica dell'Italia Centrale

Firenze

\title{
Ermenegildo Florit e la Dei Verbum
}

Ho compiuto il percorso del mio studio, scegliendo l'articolazione temporale attraverso il periodo che si snoda tra la fine del 1962 (oltre cinquant'anni fa) e la fine del 1965. Non è facile districare tutta la complessa materia in questione. Per questo rimando alla bibliografia esistente, ${ }^{1}$ ma lasciando che un posto di rilievo lo abbia lo scambio epistolare che arcivescovo di Firenze di allora, mons. Ermenegildo Florit, ${ }^{2}$ poi cardinale, ebbe con il P. Umberto Betti, e soprattutto le annotazioni che questi scrisse nel suo diario, appunto riferito ai giorni del Concilio, che si stende però fino al Natale 1978, un anno dopo che Florit era diventato arcivescovo emerito nella sede fiorentina, ${ }^{3}$ con la nomina del cardinale Giovanni Benelli (1921-1992).

\footnotetext{
${ }^{1}$ Cf. U. Betti, La dottrina del Concilio Vaticano II sulla trasmissione della rivelazione, Roma 1985²; U. Betti et al., Commento alla Costituzione dogmatica sulla Divina Rivelazione «Dei Verbum», Massimo, Milano 1967²; U. Betti, Storia della Costituzione dogmatica «Dei Verbum», in A. Favale, edd., La Costituzione Dogmatica sulla Divina Rivelazione, Magistero Conciliare, Elle Di Ci, Torino Leumann 1967, 14-152; U. Betti, La Rivelazione divina nella Chiesa, Città Nuova, Roma 1970; R. Burigana, La Bibbia nel Concilio. La redazione della costituzione «Dei Verbum» del Vaticano II, Il Mulino, Bologna 1998; ID., «Il magistero episcopale tra Roma e Firenze. La partecipazione di Ermenegildo Florit al concilio Vaticano II», in Il vescovo fra storia e teologia. Saggi in onore del card. Silvano Piovanelli, in «Vivens Homo» 11(2000), 263-300cf. S. Tarocchi, Sfogliando il Diario di Umberto Betti. Ermenegildo Florit e la Dei Verbum, «Vivens Homo» 24(2012), 77-92; anche A. Drigani, Dilexi Ecclesiam. Il cardinale Umberto Betti. Un teologo canonista, «Vivens Homo» 21(2010), 585-595. Ringrazio qui il prof. G. Aranci, docente alla FTIC e attuale vicepreside, per le preziose indicazioni che mi ha dato, e anche per l'occasione di ripercorrere con questo studio il cammino faticoso e irreversibile della Costituzione dogmatica sulla Divina Rivelazione.

${ }^{2}$ Ermenegildo Florit (Fagagna, 5 luglio 1901 - Firenze 1'8 dic. 1985). Per una biografia si veda (a cura di B.B. Camaiani) http://www.treccani.it/enciclopedia/ermenegildo-florit_(DizionarioBiografico)/ [16 maggio 2014].

${ }^{3}$ U. Betti, Diario del Concilio. 11 ottobre 1962-Natale 1978, Oggi e Domani II, 24, EDB, Bologna 2003.
} 
1962

Il mese di novembre 1962 può essere considerata una data particolarmente importante per la costituzione dogmatica sulla Divina Rivelazione, conosciuta come Dei Verbum. Il 22 novembre, infatti, si ebbe la votazione sullo schema $D e$ fontibus revelationis, già presentato all'aula il 14 novembre, per vedere «fino a che punto il giudizio negativo espresso dai padri che avevano parlato fosse condiviso anche da quelli che ancora non s'eran fatti sentire»: ${ }^{4}$ su 2209 votanti, risultavano 822 non placet; 1368 placet.

Lo schema veniva così giudicato irriformabile, e andava sostituito con un totalmente nuovo, senza il timore di irriverenza verso il romano pontefice ma per amore della chiesa. Questi numeri tuttavia non raggiungono la maggioranza qualificata dei due terzi, necessaria per respingere lo schema. Solo l'intervento di Giovanni XXIII poté demandare ad una commissione speciale la revisione dello schema preparatorio, seguente alla decisione più importante: soprassedere alla discussione dello schema preparatorio. ${ }^{5}$ Viene costituita una commissione, la "commissione mista", affidata alla presidenza dei cardinali Ottaviani (Commissione dottrinale) e Bea (Segretariato per l'unità dei cristiani), con 6 cardinali nominati dal papa (i cardinali Liénart, Frings, Ruffini, Quiroga y Palacios, Lefèbvre, Meyer) e i membri dei due organismi. ${ }^{6} \mathrm{La}$ "commissione mista" è a sua volta divisa in cinque sottocommissioni, una per ciascuno dei capitoli da rifare. ${ }^{7}$ In una serie di riunioni tra il 25 novembre e il 7 dicembre si raggiunge un accordo di massima sul nuovo schema.

1963

\section{Febbraio}

Una sottocommissione composta di quattro padri Charue, vescovo di Namur, e Florit (commissione dottrinale), Jaeger e De Smedt (Segretariato per l'unità dei cristiani) dovrà risolvere la questione del rapporto fra Scrittura e Tradizione. ${ }^{8}$ Burigana annovera Florit come «esponente di primo piano della teologia romana prima della sua nomina episcopale, assai vicino alle posizioni di Ottaviani». ${ }^{9}$

È messo a votazione (23 febbraio) il principio che nella composizione del testo si evitino espressioni che dicano o neghino che la Tradizione ha estensione

\footnotetext{
${ }^{4}$ U. Betti, Costituzione, op.cit., 32.

${ }^{5}$ U. Betti, Costituzione, op.cit., 33; cf. R. Burigana, La Bibbia nel Concilio, op.cit., 105-169.

${ }^{6}$ Cf. R. Burigana, La Bibbia nel Concilio, op.cit., 171-253.

${ }^{7}$ U. Betti, Costituzione, op.cit., 32-33.

${ }^{8}$ Cf. R. Burigana, La Bibbia nel Concilio, op.cit., 209.210.

${ }^{9}$ Cf. R. Burigana, La Bibbia nel Concilio, op.cit., 256; cf. ibid. n. 4, con bibliografia di approfondimento.
} 
più ampia della Scrittura. Il risultato dava una maggioranza schiacciante sull'approvazione del principio, ma prevalse il disaccordo sulla modalità per raggiungere concretamente quanto auspicato dal voto. Betti cita anche il vescovo di Losanna, Charriere: «che cosa si può fare se i due presidenti sono discordi?». ${ }^{10}$ Ebbe la meglio, nella provvidenziale circostanza che lo schema non venisse discusso nel secondo periodo del Concilio (29 settembre-4 dicembre 1963), il tentativo compromissorio di riprendere le opposte tendenze, già manifestatesi. ${ }^{11}$

\section{Giugno 1963}

La sera del 3 muore Giovanni XXIII. Il 21 viene eletto papa Paolo VI.

\section{6-27 settembre: Firenze - Villa Cancelli}

Florit riunisce a Firenze (a Villa Cancelli, dalle suore Orsoline di San Carlo) un gruppo d'italiani e di francesi, con il card. Lefèbvre (Bourges) e il vescovo Garrone (Tolosa). Ci saranno anche Baldassarri (Ravenna) e Carli (Segni). Lercaro, pur invitato, è totalmente contrario all'incontro.

Il Betti nota con feroce ironia che il vescovo di Segni, parlava «con voce obnubilata, come se avesse la gola ingombrata da grosse lische di pesce». ${ }^{12}$ Tuttavia, inaspettatamente, approvò la risoluzione. ${ }^{13}$ P. Betti e R. Etchegaray sono i periti rispettivi. Di fronte alle difficoltà incontrate dallo schema de revelatione, si sostiene la tesi dell'incorporazione del De divina revelatione nel De ecclesia (in mysterio Ecclesiae posita). Il Betti racconta di una provvidenziale e benefica sosta a Fiesole, dal vescovo Bagnoli e poi al convento di S. Francesco. ${ }^{14}$

\section{9 settembre}

Paolo VI inaugura il II periodo del Concilio. Il 4 dicembre lo stesso papa dissiperà le preoccupazioni sostenute nella sessione informale fiorentina, riecheggiate negli interventi di Florit (30 settembre) e di Garrone (1 ottobre).

\section{2 ottobre}

All'interno della Commissione dottrinale viene costituita una sottocommissione generale (in seguito diventerà "centrale" su impulso di Florit), di cui fanno

\footnotetext{
${ }^{10}$ Cf. U. Betti, Costituzione, op.cit., 35 n. 38.

${ }^{11} \mathrm{U}$. Betti, Costituzione, op.cit., 38.

${ }^{12}$ U. Betti, Diario del Concilio, op.cit., 22.

${ }^{13}$ Cf. anche R. Burigana, La Bibbia nel Concilio, op.cit., 239.

${ }^{14}$ Cf. U. Betti, Diario del Concilio, op.cit., 21.
} 
parte il card. Browne che la presiede, e poi Garrone, Florit, Charue e Spanedda (Bosa). Periti sono Philips, Gagnebet, Schauf e Medina. Vi sarà integrato lo stesso P. Betti, insieme a Moeller. ${ }^{15}$

\section{8 ottobre}

Florit entra a far parte della sottocommissione V, costituita all'interno della Commissione Dottrinale per rivedere i nn. 16-21 (de collegio e ministeriis Episcoporum) dello schema sulla Chiesa. Ne fanno parte Parente, Schroeffer, Volk. P. Betti vi partecipa come perito, insieme a Philips, Carlo Colombo, Rahner, Thils, Moeller, Ratzinger, Semmerloth. Il 7 novembre la commissione eleggerà Parente come presidente e Betti sarà il segretario. ${ }^{16}$ Betti annota che già l'11 ottobre 1962, Florit gli ha chiesto diventare suo teologo personale, nonostante non fosse nel numero iniziale dei periti e nonostante il fatto che prima di allora non avessero rapporti. «Non lo conosco - scrive -, ne so chi gli abbia fatto il mio nome». ${ }^{17}$ Il rapporto è proseguito a lungo: il diario ne dà testimonianza fino al Natale 1978, quando Florit, ormai arcivescovo emerito, scrive a Betti: «nulla valgo dopo il mio ritiro». Per lungo tempo, come si può raccogliere dalla corrispondenza fra i due, cercò inutilmente di farlo ordinare vescovo, a cominciare dalla richiesta che gli invia riservatamente il 21 maggio $1966 .{ }^{18}$

\section{4}

\section{4 gennaio}

Nella sottocommissione V, Garrone, con l'avallo di Florit vuole inserire un riferimento alla Tradizione nella Lumen Gentium, secondo l'esito dell'incontro di Villa Cancelli. Garrone manterrà questa posizione, insieme ad altri italiani e francesi, pur davanti all'opposizione dell'episcopato tedesco. ${ }^{19} \mathrm{P}$. Betti fa desi-

${ }^{15}$ Cf. U. Betti, Diario del Concilio, op.cit., 22.

${ }^{16}$ Cf. U. Betti, Diario del Concilio, op.cit., 23. Del rapporto costante fra Florit e Betti (Pieve S. Stefano 7 marzo 1922 - Fiesole 1 aprile 2009), sugli schemi della divina Rivelazione e sulla Chiesa, cf. U. Betti, Diario del Concilio, op.cit., 22-24.25; cf. anche 40-42, sul ruolo diretto nel mediare fra posizioni distanti (Heuschen contro Rahner), che vide emergere anche la sua capacità dottrinale, d'intesa costante con Florit. Cf. anche A. Drigani, Dilexi Ecclesiam, op.cit., 585-595: lo studio si sofferma anche sul ruolo che il teologo Betti ebbe nella commissione per la revisione del nuovo Codice di Diritto canonico (ibid. 587).

${ }^{17}$ Cf. U. Betti, Diario del Concilio, op.cit., 15; tuttavia Paolo Ristori, suo segretario particolare, ricorda cha la scelta gli fu consigliata dal Cardinale Ferdinando Giuseppe Antonelli (1896-1993), cf. M. Mancini, G. Pallanti, La preghiera spezzata. I cattolici fiorentini nella seconda metà del ' 900 , Firenze 2010.

${ }^{18}$ Cf. U. Betti, Diario del Concilio, op.cit., 212; cf 159.

${ }^{19}$ Così R. Burigana, La Bibbia nel Concilio, op.cit., 240. 
stere dal proposito, in quanto la Dei Verbum affronterà l'argomento direttamente. Betti annota con quale sottile pertinacia si cerca di criticare l'operato della sottocommissione V, sul De Ecclesia, per esempio sul ruolo del Concilio nei confronti del primato pontificio. ${ }^{20}$

\section{7 marzo}

La commissione dottrinale costituisce una sot tocommis sione (composta da sette Padri e diciannove periti: fra questi ultimi Cerfaux, Carlo Colombo - eletto vescovo lo stesso giorno; Congar, Garofalo, Grillmeier, Moeller, Rahner, Ratzinger, Rigaux, Schaf, Semmelroth). Ne è eletto presidente Charue; P. Betti ne è segretario. L'11 marzo detta sottocommissione si suddivide in due gruppi di studio (o sottocommissioni interne): il primo presieduto da Florit, si sarebbe occupato della Rivelazione e della sua trasmissione (proemio e capitolo I). Il secondo, presieduto da Charue, si occupava della parte riguardante la Scrittura (capp. II-IV). ${ }^{21}$

Sul capitolo I, e sul rapporto Scrittura - Tradizione, vennero fatte due relazioni da Schaf e da Rahner, indipendenti l'una dall'altra. Rahner e Congar prepareranno due testi nuovi (parziali) su Scrittura e Tradizione. Il lungo voto di Florit (datato Firenze, 18 gennaio) entrò in uno dei due testi su tutto il capitolo preparato dallo stesso Betti. ${ }^{22}$

Si nota in primo luogo l'insistenza sulla trasmissione della Divina Rivelazione, mediante Scrittura e Tradizione. ${ }^{23}$ Dove il n. 1 dello schema accenna alla conoscenza naturale di Dio, Florit sostiene l'insufficienza di detta affermazione, sostenendo il riferimento al Vaticano I, che tratta compiutamente della cosa. Si trasferirà l'affermazione al n. $5 .^{24}$

Un altro argomento che ebbe una stesura laboriosa fu, nel n. 2, la precedenza del termine gestis rispetto a verbis, ${ }^{25}$ con la contrapposizione fra Tromp e Rah-

${ }^{20}$ U. Betti, Diario del Concilio, op.cit., 32-33.

${ }^{21}$ Cf. U. Betti, Diario del Concilio, op.cit., 35. Lo stesso Betti riferisce le confidenze di Florit, a riguardo di attacchi contro l'ortodossia di LG 22 alla CEI (riunione del 14-16 aprile 1964).

${ }^{22}$ Sul cap. II lavoreranno insieme Semmelroth, Grillmeier e Ratzinger; sul III, Kerrigan; sul IV, da Rigaux e Turrado (testi indipendenti); sul V, congiuntamente da Semmelroth e Grillmeier). Cf. U. Betti, Costituzione, op.cit., 41 n. 49.

${ }^{23}$ Sul contenuto, cf. la sintesi di U. Betti, Costituzione, op.cit., 42.

${ }^{24}$ DV 5: «A Dio che rivela è dovuta "l'obbedienza della fede» ( $R m$ 16,26 cfr. Rm 1,5, 2 Co 10,5-6), con la quale l'uomo gli si abbandona tutt'intero e liberamente prestandogli "il pieno ossequio dell'intelletto e della volontà" e assentendo volontariamente alla Rivelazione che egli fa. Perché si possa prestare questa fede, sono necessari la grazia di Dio che previene e soccorre e gli aiuti interiori dello Spirito Santo, il quale muova il cuore e lo rivolga a Dio, apra gli occhi dello spirito e dia "a tutti dolcezza nel consentire e nel credere alla verità". Affinché poi l'intelligenza della Rivelazione diventi sempre più profonda, lo stesso Spirito Santo perfeziona continuamente la fede per mezzo dei suoi doni».

${ }^{25}$ U. Betti, Diario del Concilio, op.cit., 39. 
ner. Momentaneamente (22 aprile) prevale la formula verbis gestisque, ma nella riunione del 24 aprile una votazione della plenaria (con le due sottocommissioni congiunte) sceglie definitivamente la formula (gestis verbisque), e la tesi di $\mathrm{Bu}$ tler che mette al primo posto $\mathrm{i}$ «gesti» prima che le «parole»: così avremo nel testo definitivo revelationis oeconomia fit gestis verbisque. ${ }^{26}$ Sul punto specifico Florit, che era riuscito a trovare un accordo in precedenza, si astiene; ma insieme a Rahner sostiene l'inizio della Rivelazione dopo il peccato di Adamo, contro Congar che invece vuole si parta da Abramo.

La Tradizione orale va nominata per prima perché precede la Tradizione scritta. La tradizione trasmette tutta la divina rivelazione, ma il concilio non dovrà entrare nel merito del fatto se contenga qualcosa di diverso da quello che è trasmesso dalla Scrittura. Infine si sostiene che il deposito è stato affidato dagli Apostoli a tutta la chiesa, anche se l'interpretazione autentica spetta al magistero. ${ }^{27}$

Della discussione sui capp. II-VI, Betti rileva tout court che il n. 17 (Novi Testamenti excellentia) deriva quasi per intero dall'esposto che egli stesso ha preparato per Florit. ${ }^{28}$ Un certo rilievo lo ebbe il n. $25:{ }^{29}$ Florit, insieme a Tromp

${ }^{26}$ Così DV 2: «Piacque a Dio nella sua bontà e sapienza rivelarsi in persona e manifestare il mistero della sua volontà (cfr. Ep 1,9), mediante il quale gli uomini per mezzo di Cristo, Verbo fatto carne, hanno accesso al Padre nello Spirito Santo e sono resi partecipi della divina natura (cfr. Ep 2,18, 2P 1,4). Con questa Rivelazione infatti Dio invisibile (cfr. Col 1,15, 1Tm 1,17) nel suo grande amore parla agli uomini come ad amici (cfr. Ex 33,11, Jn 15,14-15) e si intrattiene con essi (cfr. Ba 3,38), per invitarli e ammetterli alla comunione con sé. Questa economia della Rivelazione comprende eventi e parole intimamente connessi, in modo che le opere, compiute da Dio nella storia della salvezza, manifestano e rafforzano la dottrina e le realtà significate dalle parole, mentre le parole proclamano le opere e illustrano il mistero in esse contenuto. La profonda verità, poi, che questa Rivelazione manifesta su Dio e sulla salvezza degli uomini, risplende per noi in Cristo, il quale è insieme il mediatore e la pienezza di tutta intera la Rivelazione»; cf. U. Betti, Diario del Concilio, op.cit., 42.

${ }^{27}$ Sul punto, la commissione mista non aveva raggiunto un accordo (1 marzo 1963).

${ }^{28}$ DV 17: «La parola di Dio, che è potenza divina per la salvezza di chiunque crede (cfr. Rm 1,16), si presenta e manifesta la sua forza in modo eminente negli scritti del Nuovo Testamento. Quando infatti venne la pienezza dei tempi (cfr. Ga 4,4), il Verbo si fece carne ed abitò tra noi pieno di grazia e di verità (cfr. Jn 1,14). Cristo stabilì il regno di Dio sulla terra, manifestò con opere e parole il Padre suo e se stesso e portò a compimento l'opera sua con la morte, la risurrezione e la gloriosa ascensione, nonché con l'invio dello Spirito Santo. Elevato da terra, attira tutti a sé (cfr. Jn 12,32 gr.), lui che solo ha parole di vita eterna (cfr. Jn 6,68). Ma questo mistero non fu palesato alle altre generazioni, come adesso è stato svelato ai santi apostoli suoi e ai profeti nello Spirito Santo (cfr. Ep 3,4-6, gr.), affinché predicassero l'Evangelo, suscitassero la fede in Gesù Cristo Signore e radunassero la Chiesa. Di tutto ciò gli scritti del Nuovo Testamento presentano una testimonianza perenne e divina»; cf. U. Betti, Diario del Concilio, op.cit., 43.

${ }^{29}$ DV 25: «Perciò è necessario che tutti i chierici, principalmente i sacerdoti e quanti, come i diaconi o i catechisti, attendono legittimamente al ministero della parola, conservino un contatto continuo con le Scritture mediante una lettura spirituale assidua e uno studio accurato, affinché non diventi "un vano predicatore della parola di Dio all'esterno colui che non l'ascolta dentro di sé» 
e Pelletier, vorrebbero che si mitigasse l'affermazione che raccomanda la lettura della Scrittura, data la probabile pericolosità per i più semplici. Si oppone ancora Butler, abate benedettino, con la sua esperienza di convertito dall'anglicanesimo: egli vede un giovamento personale più che un danno spirituale. ${ }^{30}$

Lo schema venne rielaborato dal 20 al 25 aprile 1964: il proemio diventerà un capitolo (De ipsa Revelatione). ${ }^{31}$ Così il precedente capitolo I diventerà il II, e viene affidato a Betti come perito relatore. ${ }^{32}$ Burigana fa emergere alcune delle perplessità di Florit verso la teologia che egli definiva lovaniense, in contrapposizione a quella che poteva offrire lui con Betti. Viceversa egli era guardato con un certo sospetto, in quanto ritenuto troppo vicino alla teologia romana più tradizionale. ${ }^{33}$

\section{1-5 giugno 1964}

La commissione dottrinale prese in esame tutto il lavoro (1-5 giugno 1964) e lo approvò, senza difficoltà eccetto il testo sulla Tradizione. P. Betti nel suo Diario mette in luce l'ostilità di Franič e Schauf contro Rahner, e di Ottaviani contro Florit, assente in quella sede perché convalescente dopo un intervento alla retina. Parla di «sadico opportunismo» di Ottaviani contro l'arcivescovo di Firenze, che già alla CEI avrebbe influenzato l'episcopato italiano a riguardo della «Tradizio-

(38), mentre deve partecipare ai fedeli a lui affidati le sovrabbondanti ricchezze della parola divina, specialmente nella sacra liturgia. Parimenti il santo Concilio esorta con ardore e insistenza tutti

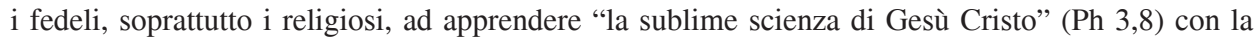
frequente lettura delle divine Scritture. "L'ignoranza delle Scritture, infatti, è ignoranza di Cristo" (39). Si accostino essi volentieri al sacro testo, sia per mezzo della sacra liturgia, che è impregnata di parole divine, sia mediante la pia lettura, sia per mezzo delle iniziative adatte a tale scopo e di altri sussidi, che con l'approvazione e a cura dei pastori della Chiesa, lodevolmente oggi si diffondono ovunque. Si ricordino però che la lettura della sacra Scrittura dev'essere accompagnata dalla preghiera, affinché si stabilisca il dialogo tra Dio e l'uomo; poiché «quando preghiamo, parliamo con lui; lui ascoltiamo, quando leggiamo gli oracoli divini”. Compete ai vescovi, «depositari della dottrina apostolica", ammaestrare opportunamente i fedeli loro affidati sul retto uso dei libri divini, in modo particolare del Nuovo Testamento e in primo luogo dei Vangeli, grazie a traduzioni dei sacri testi; queste devono essere corredate delle note necessarie e veramente sufficienti, affinché i figli della Chiesa si familiarizzino con sicurezza e profitto con le sacre Scritture e si imbevano del loro spirito. Inoltre, siano preparate edizioni della sacra Scrittura fornite di idonee annotazioni, ad uso anche dei non cristiani e adattate alla loro situazione; sia i pastori d'anime, sia i cristiani di qualsiasi stato avranno cura di diffonderle con zelo e prudenza».

${ }^{30}$ Cf. U. Betti, Diario del Concilio, op.cit., 43.

${ }^{31}$ È interessante l'annotazione di Betti (U. Betti, Diario del Concilio, op.cit., 38), che riferendosi ad una dichiarazione del Vaticano I (Mansi, 51, 127D), sostiene che non è dottrina il proemio di un documento.

${ }^{32}$ Cf. U. Betti, Costituzione, op.cit., 44 n. 54; U. Betti, Diario del Concilio, op.cit., 40-42.

${ }^{33}$ Cf. la ricostruzione offerta R. Burigana, La Bibbia nel Concilio, 263-271; 268. 
ne costitutiva senza limiti». ${ }^{34}$ Con una votazione dal risultato di 17 a 7 , il 3 giugno venne approvato, con l'intesa del maggior contenuto oggettivo della Tradizione sulla Scrittura. Fu deciso (6 giugno) che Florit fosse il rappresentante della maggioranza (con il proemio e i capp. I e II, De ipsa revelatione, De divinae revelationis transmissione). Ci saranno anche van Dodewaard (Haarlem), rappresentante della maggioranza per i rimanenti capitoli, e Franič (Spalato), come rappresentante della minoranza. Il Betti registra i suoi «segni vistosi di disgustata sofferenza». ${ }^{35}$

Da notare che il 21 aprile 1964 era stata approvata anche l'istruzione Sancta Mater Ecclesia, della Pontificia Commissione Biblica sulla storicità dei Vangeli, che dunque era conosciuta dalla sottocommissione. ${ }^{36}$

Betti evidenzia anche alcuni sotterfugi redazionali - si fa il nome dello stesso Tromp -, operati sul testo consegnato ai Padri, per diminuire il valore delle due costituzioni: la scomparsa della parola «dogmatica» dai due titoli, ed una modifica al n. 8 della Dei verbum. Ad esempio l'avverbio semel fatto diventare simul nel testo di Giuda 3: «Carissimi, avendo un gran desiderio di scrivervi riguardo alla nostra comune salvezza, sono stato costretto a farlo per esortarvi a combattere per la fede, che fu trasmessa ai santi una volta per sempre (semel)», ${ }^{37}$ per aumentare il valore della Tradizione rispetto alla Scrittura. Questo inquieta non poco Florit, che interviene presso lo stesso Tromp. ${ }^{38}$ Lo stesso Florit, riferisce ancora P. Betti, invitato da Ottaviani ad affrettare la consegna della relazione, ${ }^{39}$ vi si dedica alacremente. Betti, con molta franchezza, dice che i primi appunti ricevuti «non sono un granché». ${ }^{40}$ Il suo personale lavoro, molto denso anche per Florit in alcuni passaggi, se sente il bisogno di una terza lettura, ${ }^{41}$ è completato il 12 settembre.

${ }^{34}$ Cf. U. Betti, Diario del Concilio, op.cit., 46-47. Più avanti (p. 48) descrive Ottaviani come colui che «trasudava umor nero» ancora nei confronti di Florit. Anche R. Burigana, La Bibbia nel Concilio, op.cit., 285-286.375.

${ }^{35}$ Avrà lo stesso compito anche per il III cp. della Lumen Gentium. Cf. U. Betti, Diario del Concilio, op.cit., 75.

${ }^{36}$ Cf. R. Burigana, La Bibbia nel Concilio, op.cit., 276-282.

${ }^{37}$ Greco: $\alpha \pi \pi \xi$. Cf. DV 8: «Pertanto la predicazione apostolica, che è espressa in modo speciale nei libri ispirati, doveva esser conservata con una successione ininterrotta fino alla fine dei tempi. Gli apostoli perciò, trasmettendo ciò che essi stessi avevano ricevuto, ammoniscono i fedeli ad attenersi alle tradizioni che avevano appreso sia a voce che per iscritto (cfr. 2Th 2,15), e di combattere per quella fede che era stata ad essi trasmessa una volta per sempre (utque pro s e me $l$ sibi tradita fide decertent (cf. Jud 1,3)».

${ }^{38}$ Lettera di Florit a Betti del 25 agosto 1964; cf. U. Betti, Diario del Concilio, 51. 196; anche U. Betti, Costituzione, op.cit., 45; R. Burigana, La Bibbia nel Concilio, op.cit., 297 n. 81; 364.

${ }^{39}$ Cf. U. Betti, Diario del Concilio, op.cit., 196: è la medesima lettera di Florit del 25 agosto, ma Betti già ne parla in un appunto del 18 luglio, data a cui risale la precedente missiva dell'arcivescovo.

${ }^{40}$ Cf. U. Betti, Diario del Concilio, op.cit., 52.

${ }^{41}$ Cf. U. Betti, Diario del Concilio, op.cit., 52. 197. 


\section{0 settembre 1964}

Nel suo diario, in data 25 settembre, P. Betti riferisce che Florit «sembra turbato da ritornanti ripensamenti»; tre giorni dopo parla ancora di «qualche ritorno di tentennamenti. ${ }^{42}$ Questo stato d'animo gli fa rallentare l'arrivo in aula la mattina del 30 settembre. «Ha fatto proseguire la macchina - scrive P. Betti - verso i giardini Vaticani, e lì seduti si respirano ancora dubbi invece d'aria buona». Tanto che «arrivato il momento, legge la relazione con una voce che sembra gli fosse stata imprestata». ${ }^{43}$

Florit nel suo intervento - che ebbe peraltro un ampio consenso,$-{ }^{44}$ cercò di far notare che i capp. I e II avevano ripreso la dottrina tradizionale, arricchendola di aspetti nuovi, in particolare sulla tradizione, com'era stato notato dal Patriarca dei Maroniti.

Con molta freddezza Burigana analizza l'intervento dell'arcivescovo di Firenze, parlando di una velata critica alla commissione mista, e di conseguenza l'«inutile e pericoloso» coinvolgimento di Bea e dell'organismo da lui presieduto, ma anche del suo rispetto, quand'anche in contrasto con le sue opinioni personali, nei confronti della maggioranza dei Padri. Aggiunge poi che Florit «chiese ai Padri di votare lo schema presentato, perché costituiva un punto d'equilibrio tra le due opposte scuole teologiche, che rappresentavano gli estremi inconciliabili». Tutto questo per «scegliere una formula che esprimesse in termini essenziali la dottrina cattolica e impedisse fratture in concilio». Il senso, scrive ancora Burigana, era l'idea che il concilio «era chiamato ad approfondire e reinterpretare la storia conciliare precedente, non a rimuovere una parte di essa»..$^{45}$

Forse, va detto, basta e avanza la descrizione di Betti dell'indole estremamente indecisa di Florit sulle questioni su cui doveva esporre, ma anche la saggezza di essersi avvalso di un teologo raffinato come Betti, capace di mediare ma anche di portare il concilio su vie che alla vigilia nessuno si sarebbe atteso.

Le modifiche proposte, per restare al contributo di Florit, si concentrano sul n. 3 e il $n .4$ del cap. I (rispettivamente de evangelica revelationis praeparationis e de Christo revelationis consummatore) ${ }^{46}$ e sul n. 8 del cap. II (de sacra Tradi-

${ }^{42}$ U. Betti, Diario del Concilio, op.cit., 54; più cauto Burigana che registra l'auto-attribuzione di Betti della prima bozza della relazione di Florit (La Bibbia nel Concilio, 308, n. 100).

${ }^{43}$ U. Betti, Diario del Concilio, op.cit., 55.

${ }^{44}$ Fra i dissidenti l'indiano Attipetty, che B. Rigaux - nota il Betti non senza un sorriso «chiamava "Antibetti" per la circostanza»; cf. U. Betti, Diario del Concilio, 55. In aula, scrive Burigana (La Bibbia nel Concilio, 313 n. 108) dovette comunque destare qualche sorpresa, se anche il vescovo di Volterra, Bergonzini, scrisse nel suo diario che si era trattata di una vera conversione, la seconda dopo «Parente, alla corrente dominante nel concilio».

${ }^{45}$ R. Burigana, La Bibbia nel Concilio, op.cit., 314-316.

${ }^{46}$ DV 3-4: «Dio, il quale crea e conserva tutte le cose per mezzo del Verbo (cfr. Jn 1,3), offre agli uomini nelle cose create una perenne testimonianza di sé (cfr. Rm 1,19-20); inoltre, volendo aprire la via di una salvezza superiore, fin dal principio manifestò se stesso ai progenitori. Dopo la 
tione).$^{47}$ Ancora sul n. 9 (sulla mutua relatio fra Tradizione e Scrittura). ${ }^{48}$ Dopo gli emendamenti votati il 20 e 21 ottobre, l'intera commissione dottrinale discusse il testo il 10 e 11 novembre, approvando nella sostanza l'opera della sottocommissione. ${ }^{49}$ In quell'occasione, sostiene ancora Burigana, Florit, con un inter-

loro caduta, con la promessa della redenzione, li risollevò alla speranza della salvezza (cfr. Gn 3,15), ed ebbe assidua cura del genere umano, per dare la vita eterna a tutti coloro i quali cercano la salvezza con la perseveranza nella pratica del bene (cfr. Rm 2,6-7). A suo tempo chiamò Abramo, per fare di lui un gran popolo (cfr. Gn 12,2); dopo i patriarchi ammaestrò questo popolo per mezzo di Mosè e dei profeti, affinché lo riconoscesse come il solo Dio vivo e vero, Padre provvido e giusto giudice, e stesse in attesa del Salvatore promesso, preparando in tal modo lungo i secoli la via all'Evangelo. Dopo aver a più riprese e in più modi, parlato per mezzo dei profeti, Dio "alla fine, nei giorni nostri, ha parlato a noi per mezzo del Figlio» (He 1,1-2). Mandò infatti suo Figlio, cioè il Verbo eterno, che illumina tutti gli uomini, affinché dimorasse tra gli uomini e spiegasse loro i segreti di Dio (cfr. Jn 1,1-18). Gesù Cristo dunque, Verbo fatto carne, mandato come «uomo agli uomini" (3), "parla le parole di Dio" (Jn 3,34) e porta a compimento l'opera di salvezza affidatagli dal Padre (cfr. Jn 5,36, Jn 17,4). Perciò egli, vedendo il quale si vede anche il Padre (cfr. Jn 14,9), col fatto stesso della sua presenza e con la manifestazione che fa di sé con le parole e con le opere, con i segni e con i miracoli, e specialmente con la sua morte e la sua risurrezione di tra i morti, e infine con l'invio dello Spirito di verità, compie e completa la Rivelazione e la corrobora con la testimonianza divina, che cioè Dio è con noi per liberarci dalle tenebre del peccato e della morte e risuscitarci per la vita eterna. L'economia cristiana dunque, in quanto è l'Alleanza nuova e definitiva, non passerà mai, e non è da aspettarsi alcun'altra Rivelazione pubblica prima della manifestazione gloriosa del Signore nostro Gesù Cristo (cfr. 1 Tm 6,14 e Tt 2,13)».

${ }^{47}$ DV 8: «Pertanto la predicazione apostolica, che è espressa in modo speciale nei libri ispirati, doveva esser conservata con una successione ininterrotta fino alla fine dei tempi. Gli apostoli perciò, trasmettendo ciò che essi stessi avevano ricevuto, ammoniscono i fedeli ad attenersi alle tradizioni che avevano appreso sia a voce che per iscritto (cfr. 2 Th 2,15), e di combattere per quella fede che era stata ad essi trasmessa una volta per sempre (11). Ciò che fu trasmesso dagli apostoli, poi, comprende tutto quanto contribuisce alla condotta santa del popolo di Dio e all'incremento della fede; così la Chiesa nella sua dottrina, nella sua vita e nel suo culto, perpetua e trasmette a tutte le generazioni tutto ciò che essa è, tutto ciò che essa crede (cunctisque generationibus transmittit omne quod ipsa est, omne quod credit). Questa Tradizione di origine apostolica progredisce nella Chiesa con l'assistenza dello Spirito Santo: cresce infatti la comprensione, tanto delle cose quanto delle parole trasmesse, sia con la contemplazione e lo studio dei credenti che le meditano in cuor loro (cfr. Lc 2,19, Lc 2,51), sia con la intelligenza data da una più profonda esperienza delle cose spirituali, sia per la predicazione di coloro i quali con la successione episcopale hanno ricevuto un carisma sicuro di verità. Così la Chiesa nel corso dei secoli tende incessantemente alla pienezza della verità divina, finché in essa vengano a compimento le parole di Dio.

Le asserzioni dei santi Padri attestano la vivificante presenza di questa Tradizione, le cui ricchezze sono trasfuse nella pratica e nella vita della Chiesa che crede e che prega. È questa Tradizione che fa conoscere alla Chiesa l'intero canone dei libri sacri e nella Chiesa fa più profondamente comprendere e rende ininterrottamente operanti le stesse sacre Scritture. Così Dio, il quale ha parlato in passato non cessa di parlare con la sposa del suo Figlio diletto, e lo Spirito Santo, per mezzo del quale la viva voce dell'Evangelo risuona nella Chiesa e per mezzo di questa nel mondo, introduce i credenti alla verità intera e in essi fa risiedere la parola di Cristo in tutta la sua ricchezza (cfr. Col 3,16)».

${ }^{48}$ DV 9: «La sacra Tradizione dunque e la sacra Scrittura sono strettamente congiunte e comunicanti tra loro. Poiché ambedue scaturiscono dalla stessa divina sorgente, esse formano in certo qual modo un tutto e tendono allo stesso fine. Infatti la sacra Scrittura e a parola di Dio in quanto 
vento vigoroso (così scrisse lo stesso Semmelroth) «dichiarò la sua contrarietà ad un nuovo coinvolgimento del segretariato nell'elaborazione del testo, perché questo significava distruggere l'equilibrio, raggiunto su una formula che riassumeva la dottrina cattolica sulla tradizione, teneva conto delle osservazioni dei Padri e lasciava aperta la questione non pronunciando condanne». Per questo «si faceva garante dell'ortodossia ... respingendo le critiche di debolezza dottrinale, criptoprotestantesimo e modernismo»..$^{50}$

La minoranza che «incominciava a dar segni di una certa accondiscendenza», scrive il P. Betti, rinunziò al momento di designare un proprio relatore. ${ }^{51}$

Burigana ha tuttavia qualche dubbio sulla decisione decisione: «fu solo la mancanza di tempo ad impedire una votazione sullo schema (scil. in aula conciliare) oppure si preferì rinviare questa all'ultimo periodo per lasciare ampio margine alla discussione durante l'intersessione? La minoranza conciliare ... avrebbe presto dimostrato che non aveva abbandonato l'idea di intervenire ancora». ${ }^{52}$

Il 20 novembre lo Schema fu distribuito ai Padri. Ritornerà in aula nel quarto periodo del Concilio, che avrà inizio il 14 settembre 1965.

\section{5}

\section{Febbraio-agosto 1965}

In una lettera a P. Betti, Florit ricorda «uno stampato» diffuso «dai vescovi di Toscana» fra i «Padri conciliari italiani», a firma di «Carli e Perantoni», «non favorevole allo schema attuale». ${ }^{53}$ Nelle settimane successive Florit sembra preoccupato di dare in qualche maniera ascolto a queste preoccupazioni, ${ }^{54}$ come anche al documento che esisterebbe al Pontificio Istituto Biblico, secondo Ottaviani. ${ }^{55}$ Il P. Betti annota: «vado verso la convinzione che sia meglio astenersi da ogni intervento, per non dare importanza a voci stonate». ${ }^{56}$

consegnata per iscritto per ispirazione dello Spirito divino; quanto alla sacra Tradizione, essa trasmette integralmente la paro a di Dio - affidata da Cristo Signore e dallo Spirito Santo agli apostoli - ai loro successori, affinché, illuminati dallo Spirito di verità, con la loro predicazione fedelmente la conservino, la espongano e la diffondano; ne risulta così che la Chiesa attinge la certezza su tutte le cose rivelate non dalla sola Scrittura e che di conseguenza l'una e l'altra devono essere accettate e venerate con pari sentimento di pietà e riverenza».

${ }^{49}$ Cf. R. Burigana, La Bibbia nel Concilio, op.cit., 354-361.

${ }^{50}$ R. Burigana, La Bibbia nel Concilio, op.cit., 358.

${ }^{51}$ Così U. Betti, Costituzione, op.cit., 53.

${ }^{52}$ R. Burigana, La Bibbia nel Concilio, op.cit., 360-361; anche 374-377.

${ }^{53}$ La data: 8 febbraio 1965; cf. U. Betti, Diario del Concilio, op.cit., 62.201.

${ }^{54}$ Biglietto senza data citato nel diario di Betti (12 aprile) e lettera di Florit del 20 aprile; U. Betti, Diario del Concilio, op.cit., 64-65.200-202. Secondo i testi citati se ne starebbero preoccupando anche Ratzinger e Congar. Cf. R. Burigana, La Bibbia nel Concilio, op.cit., 371 n. 15.

${ }^{55}$ U. Betti, Diario del Concilio, op.cit., 66.203; cf. R. Burigana, La Bibbia nel Concilio, op.cit., 372 n. 17.

${ }^{56}$ U. Betti, Diario del Concilio, op.cit., 66. Di fatto i documenti registrano una breve visita di 
Successivamente, prima Florit, quindi Charue, Colombo e Philips, prendono posizione contro il "Comitato internazionale". Annota il P. Betti che «non è conveniente prender posizione ufficiale nei confronti dell'azione turbolenta promossa dal Comitato internazionale». Solo quando Carli scrive al papa, chiedendo una nuova discussione sullo schema De Divina Revelatione, il cardinale segretario di Stato, Amleto Cicognani, rispose di non approvare l'esistenza del "Comitato", e le sue imprese erano biasimevoli. ${ }^{57}$

Burigana sottolinea che, secondo Florit, non si dovevano neanche «sottovalutare le voci, che (scil. contrariamente al previsto) chiedevano la presentazione di una relazione di minoranza». Ma faceva affidamento sul consenso sostanziale dei teologi di Lovanio, trascurando però - dice ancora Burigana - che il parere favorevole precedentemente espresso era dettato solo dal timore di un insabbiamento dello schema nella commissione dottrinale. ${ }^{58}$

L'arcivescovo di Firenze in quella fase delicata - annota Burigana - si mosse spendendo il «prestigio che era venuto acquisendo verso i settori più attenti al rinnovamento dell'esegesi nei mesi della sua copresidenza alla sottocommissione, ma al tempo stesso mostrava attenzione nei confronti delle richieste del papa e della minoranza conciliare». ${ }^{59}$ Era necessario proporre una formula che ottenesse un'ampia maggioranza, evitando stravolgimenti delle minoranze, anche senza accogliere le esigenze delle ali estreme dei due schieramenti, soprattutto il rischio che il Concilio si chiudesse senza la costituzione sulla Rivelazione.

Nel frattempo il P. Betti intervenne all'assemblea generale della CEI, alla Domus Mariae, a proposito di due "modi", ${ }^{60}$ che integrano il n. 8 dello schema, «per indicare l'importanza che, a titolo speciale, ha il magistero nel progresso della tradizione»; e il n. 9, perché si comprenda meglio che «dalla Scrittura sola non si può comprendere tutto ciò che è stato rivelato»». ${ }^{61}$ Indecifrabile la reazione dei presenti - annota il P. Umberto - ma «quella dei cardinali Ruffini e Siri mi è sembrata un'impressione poco buona». ${ }^{62}$ Niente di più vero.

Lefèbvre [Betti annota con un significativo “!”] a Firenze il 10 settembre 1965, concludendo che «la ristrettezza del tempo gli permise solo di salutarmi» (scil. Florit) (U. Betti, Diario del Concilio, op.cit., 67.205).

${ }^{57}$ U. Betti, Diario del Concilio, op.cit., 68. Altri, molto più pragmaticamente, sostengono che non si sconfessasse il contenuto della proposta - la richiesta di una relazione di minoranza - ma le modalità della richiesta.

${ }^{58}$ R. Burigana, La Bibbia nel Concilio, op.cit., 375-376.

${ }^{59}$ R. Burigana, La Bibbia nel Concilio, op.cit., 387.

${ }^{60}$ R. Burigana (La Bibbia nel Concilio, op.cit., 388) aggiunge un terzo e quarto modo sui nn. 11 e 25 .

${ }^{61}$ U. Betti, Diario del Concilio, op.cit., 68-69.

${ }^{62}$ U. Betti, Diario del Concilio, op.cit., 69; su un intervento di Siri, che riprende i temi di Carli, ma coinvolgendo Ottaviani e Cicognani, ha anche l'avallo di Paolo VI, cf. R. Burigana, La Bibbia nel Concilio, op.cit., 386. 


\section{0-30 settembre 1965}

Nella definizione del calendario intervenne la commissione dottrinale. Tromp e Philips propendevano per una votazione preliminare, mentre Florit, forzando il regolamento, chiedeva che la relazione precedesse la votazione preliminare. Se fosse stata accettata questa tesi, ciò avrebbe permesso alla minoranza di avanzare un'altra volta la proposta di una relazione sulla tradizione. ${ }^{63}$

La proposta di Florit non venne accolta e il 17 settembre $^{64}$ venne distribuito il calendario delle votazioni. Lo stesso giorno viene approvato il I capitolo con il seguente risultato: votanti 2079; placet 1822 ; non placet 3 ; placet iuxta modum 248; nulli 9. Il II capitolo ${ }^{65}$ viene approvato il 21 settembre con il risultato che segue: votanti 2246; placet 1874; non placet 9; placet iuxta modum 354; nulli 9. Dopo l'approvazione degli altri capitoli, si può notare che il cap. II ha il numero più alto di placet iuxta modum: 354, contro i 248 del I capitolo, i 324 del III capitolo, i 47 del IV - l'unico che non ebbe neanche un non placet -, i 313 del V e i 212 del VI, per un totale di 1498 placet iuxta modum.

Com'è noto, la cifra in questione indica il numero delle votazioni, non quello dei Padri che così avevano votato, né quello degli emendamenti richiesti. «Il numero dei Padri è molto inferiore, dato che non pochi di essi dettero la stessa risposta per diversi capitoli; è molto inferiore anche il numero degli emendamenti, perché gran parte sono indicazione comune di più votazioni». ${ }^{66}$ Così Betti poté scrivere nel suo diario che l'alto numero dei placet iuxta modum «avrebbe dato ancora lavoro, ma senza pericoli di dirottamento». ${ }^{67}$

Alla classificazione dei «modi» attese un gruppo ristretto, la c o $\mathrm{m} \mathrm{m}$ is s i o ne tecnica. Ancora una volta la preside Charue, a nome di Ottaviani e ne fanno parte, per i capp. I-II, il neocardinale Florit (22 febbraio 1965) e, per i capp. III-VI, van Dodewaard (Haarlem). Oltre al segretario della s ot to c o m m i s si on e Betti e tre altri (il vescovo Heuschen, i due segretari della Commissione dottrinale Tromp e Philips), sempre presenti alle riunioni, venivano convocati di volta il volta i periti relatori dei singoli capitoli. La commissione concluse i lavori del cap. I tra il 22 e il 23 settembre, e il cap. II tra il 23 e il 25 settembre. I lavori si concludono entro il 30 settembre per gli altri capitoli. ${ }^{68}$

${ }^{63}$ Cf. R. Burigana, La Bibbia nel Concilio, op.cit., 389-390.

${ }^{64}$ Così U. Betti, Diario del Concilio, op.cit., 69. Secondo R. Burigana, La Bibbia nel Concilio, op.cit., 390 è il 20 settembre, ma evidentemente si tratta di un refuso.

${ }^{65}$ Non venne letta da Florit la relazione ufficiale, «perché i Padri l'avevano ricevuta insieme al testo il 20 novembre 1964» (U. Betti, Diario del Concilio, op.cit., 69).

${ }^{66}$ U. Betti, Costituzione, op.cit., 55.

${ }^{67}$ U. Betti, Diario del Concilio, op.cit., 69.

${ }^{68}$ Cf. R. Burigana, La Bibbia nel Concilio, op.cit., 392. 


\section{1-19 ottobre 1965}

Da questi lavori risultò che $\mathrm{i}$ «modi» raggruppati secondo lo schema erano 212: 39 per il cap. I e 60 per il II. ${ }^{69} \mathrm{Il}$ P. Betti, nello specifico, pose l'attenzione ai due modi già presentati all'episcopato italiano. L'aggiunta al n. 8 veniva richiesta da 175 Padri; al seconda aggiunta richiesta da 111 Padri. Pur con qualche complicazione sostenuta da Tromp, che si avvale di una lettera di Felici che pretende di rappresentare il pensiero del papa, ma altri sostengono che «se il papa la pensa davvero così, avrà autorità e modi per farlo sapere con tutta chiarezza». ${ }^{70}$ Tromp riuscì però a far approvare un'aggiunta al n. 9 (directe probari) che poteva essere interpretata nel senso che «ogni verità può essere dimostrata dalla Scrittura indirettamente per il solo fatto che attesta l'esistenza di un magistero infallibile... di modo che il magistero potrebbe definire una verità senza nessun riferimento alla Scrittura, e sarebbe tuttavia divinamente rivelata». ${ }^{71}$ Nonostante voci come Fernandez, Gagnebet e Parente, il 1 ottobre, in Commissione dottrinale, quest'aggiunta venne respinta: Betti descrive Tromp come «comprensibilmente handicappato. E palesemente irritato, anche con me». ${ }^{72} \mathrm{Si}$ può concludere, come fa altrove, che «la lunga covatura era servita soltanto a farne un uovo barlaccio». ${ }^{73}$ Tuttavia, dopo l'approvazione finale in aula della Dei Verbum, fu probabilmente ancora Tromp a sostituire communicantes con communicans, ${ }^{74}$ con il risultato che «chi comunica i doni divini è Cristo, e non più gli Apostoli». Le reazioni, registrate con freddezza da perito settore, dallo stesso Betti: Charue rimase «livido»; Florit restò «allibito»; Ottaviani tenne un' «imperturbabilità olimpica». Felici, pur chiamato in causa, non dette mai una rettifica pubblica. ${ }^{75}$ Questa si ebbe solo il 17 dicembre a Concilio ormai concluso. ${ }^{76}$

L'intera commissione dottrinale prese in esame tutto il testo, non senza un intervento diretto di Paolo VI (18 ottobre), ${ }^{77}$ che invitò a riconsiderare i nn. 9 (la

${ }^{69}$ Cf. U. Betti, Costituzione, op.cit., 56.

${ }^{70}$ Cf. R. Burigana, La Bibbia nel Concilio, op.cit., 392-393.

${ }^{71}$ U. Betti, Diario del Concilio, op.cit., 70.

${ }^{72}$ U. Betti, Diario del Concilio, op.cit., 72-73.

${ }^{73}$ U. Betti, Diario del Concilio, op.cit., 73.

${ }^{74}$ Cf. DV 7: «Dio, con somma benignità, dispose che quanto egli aveva rivelato per la salvezza di tutte le genti, rimanesse per sempre integro e venisse trasmesso a tutte le generazioni. Perciò Cristo Signore, nel quale trova compimento tutta intera la Rivelazione di Dio altissimo, ordinò agli apostoli che l'Evangelo, prima promesso per mezzo dei profeti e da lui adempiuto e promulgato di persona venisse da loro predicato a tutti come la fonte di ogni verità salutare e di ogni regola morale, comunicando (communicantes) così ad essi i doni divini».

${ }^{75}$ U. Betti, Diario del Concilio, op.cit., 82.83.

${ }^{76}$ Cf. R. Burigana, La Bibbia al Concilio, op.cit., 433-434.

${ }^{77}$ In concomitanza con un udienza a Charue e a Florit (R. Burigana, La Bibbia nel Concilio, op.cit., 416; anche 415-430). Secondo lo studioso, l'atteggiamento tenuto da Florit in seguito divise definitivamente i due esponenti conciliari (420). 
tradizione costitutiva), 11 (1' "inerranza" e la veritas salutaris) $)^{78}$ e 19 (la storicità dei vangeli). ${ }^{79}$

Questo avvenne il 19 ottobre. Al termine di una giornata convulsa, alla presenza di un gruppo ristretto della commissione dottrinale di Ottaviani, Charue, Browne, Tromp e Philips, oltre allo stesso Florit - di cui si riferisce una riflessione, tratta dal diario, sul ruolo egemonico di Bea, che il papa aveva aggiunto, sua sponte, allo stesso gruppo -, annota P. Betti che finalmente «s'era arrivati in porto con tanto ritardo, quando si poteva arrivarci molto prima e senza scomodare il papa». ${ }^{80}$

Il 29 di settembre venne introdotto il proemio con il celebre incipit: Dei Verbi religiose audiens et fideliter proclamans, al posto del precedente Sanctissima Synodus, troppo simile a Sacrosanctum Concilium. Del cap. I (esaminato lo stesso giorno), ${ }^{81}$ si richiama qui il n. 3 sulla differenza tra rivelazione naturale e quel-

${ }^{78}$ DV 11: "Le verità divinamente rivelate, che sono contenute ed espresse nei libri della sacra Scrittura, furono scritte per ispirazione dello Spirito Santo La santa madre Chiesa, per fede apostolica, ritiene sacri e canonici tutti interi i libri sia del Vecchio che del Nuovo Testamento, con tutte le loro parti, perché scritti per ispirazione dello Spirito Santo (cfr. Jn 20,31, 2Tm 3,16); hanno Dio per autore e come tali sono stati consegnati alla Chiesa per la composizione dei libri sacri, Dio scelse e si servì di uomini nel possesso delle loro facoltà e capacità, affinché, agendo egli in essi e per loro mezzo, scrivessero come veri autori, tutte e soltanto quelle cose che egli voleva fossero scritte.

Poiché dunque tutto ciò che gli autori ispirati o agiografi asseriscono è da ritenersi asserito dallo Spirito Santo, bisogna ritenere, per conseguenza, che i libri della Scrittura insegnano con certezza, fedelmente e senza errore (firmiter, fideliter et sine errore) la verità che Dio, per la nostra salvezza, volle fosse consegnata nelle sacre Scritture (veritatem, quam Deus nostrae salutis causa Litteris Sacris consignari voluit). Pertanto «ogni Scrittura divinamente ispirata è anche utile per insegnare, per convincere, per correggere, per educare alla giustizia, affinché l'uomo di Dio sia perfetto, addestrato ad ogni opera buona».

${ }^{79}$ DV 19: «La santa madre Chiesa ha ritenuto e ritiene con fermezza e con la più grande costanza che i quattro suindicati Vangeli, di cui afferma senza esitazione la storicità, trasmettono fedelmente quanto Gesù Figlio di Dio, durante la sua vita tra gli uomini, effettivamente operò e insegnò per la loro eterna salvezza, fino al giorno in cui fu assunto in cielo (cfr Ac 1,1-2). Gli apostoli poi, dopo l'Ascensione del Signore, trasmisero ai loro ascoltatori ciò che egli aveva detto e fatto, con quella più completa intelligenza delle cose, di cui essi, ammaestrati dagli eventi gloriosi di Cristo e illuminati dallo Spirito di verità, godevano. E gli autori sacri scrissero i quattro Vangeli, scegliendo alcune cose tra le molte che erano tramandate a voce o già per iscritto, redigendo un riassunto di altre, o spiegandole con riguardo alla situazione delle Chiese, conservando infine il carattere di predicazione, sempre però in modo tale da riferire su Gesù cose vere e sincere. Essi infatti, attingendo sia ai propri ricordi sia alla testimonianza di coloro i quali "fin dal principio furono testimoni oculari e ministri della parola", scrissero con l'intenzione di farci conoscere la "verità" (cfr. Lc 1,2-4) degli insegnamenti che abbiamo ricevuto».

${ }^{80}$ Cf. U. Betti, Diario del Concilio, op.cit., 77-78; anche R. Burigana, La Bibbia nel Concilio, op.cit., 424-428.

${ }^{81}$ Cf. R. Burigana, La Bibbia nel Concilio, op.cit., 395. Lo stesso studioso nota che tale scelta «indica i limiti dello schema redatto», perché fa pensare ad un testo sulla Parola di Dio, mentre tratta il tema della Rivelazione, in forte continuità con la fase preparatoria. 
la soprannaturale; e il n. 4, sul Cristo pienezza della Rivelazione. ${ }^{82}$ Del cap. II (esaminato il 1, il 4, il 6 e il 19 ottobre), ${ }^{83}$ si richiama il n. 8: il magistero, come fattore del progresso della Tradizione $;{ }^{84}$ e, ancora, il n. 9, che dopo una laboriosa discussione, vede questa aggiunta: quo fit ut Ecclesia certitudinem sumat de omnibus revelatis non per solam Sacram Scripturam hauriat. ${ }^{85}$ Senza cambiare la sostanza, si afferma che la Scrittura senza la Tradizione non basta a dare la certezza di tutte le verità rivelate. «Ma non è detto che, pur non escludendolo, che quel che la Scrittura non è sufficiente a farci conoscere da sola sia la sola Tradizione a farcelo conoscere. Dal testo così emendato non si può concludere né che la Scrittura è la codificazione di tutta la Rivelazione né che la Tradizione è un supplemento quantitativo della Scrittura». ${ }^{86}$ Il cap. III fu esaminato il 4, il 6 e il 19 ottobre. Di esso si richiama qui il n. 19 e la questione della "veritatem salutarem", risolta brillantemente con l'inserimento dell'inciso seguente: veritatem, quam Deus nostrae salutis causa, litteris sacris consignari voluit. La «frettolosa traduzione dell'Osservatore Romano (22-23 novembre 1965 p. 3 col. 4) non compresse che «causa» non è nominativo ma ablativo, come un buono studente del liceo dovrebbe sapere...». ${ }^{87} \mathrm{Da}$ allora in poi secondo la richiesta di Charue, l'edizione latina scriverà causà. Il cap. IV venne esaminato il 6 ottobre; il V il 6, il 9 e il 19 ottobre (si richiama qui dal n. 19, la questione della storicità, risolta con l'inciso quorum historicitatem incunctanter affirmat); 88 il VI fu esaminato il 9 e l'11 ottobre.

\section{9 ottobre 1965}

Prima della votazione finale, fissata per il 29 ottobre 1965, ci fu solo un estremo tentativo di resistenza, su un foglio fatto circolare dal "Comitato episcopa-

\footnotetext{
${ }^{82}$ Cf. sopra, n. 46.

${ }^{83}$ Sul complesso travaglio di quei giorni, compreso un nuovo ipotizzato intervento di Paolo VI, che agitò non poco Florit, «sempre più preoccupato per l'ortodossia» dello schema sulla rivelazione», cf. R. Burigana, La Bibbia nel Concilio, op.cit., 398-400.402-415.

${ }^{84}$ Cf. sopra, n. 47.

${ }^{85}$ Cf. sopra, n. 48.

${ }^{86}$ U. Betti, Costituzione, op.cit., 61; anche U. Betti, Diario del Concilio, op.cit., 73-75.

${ }^{87}$ Cf. U. Betti, Costituzione, op.cit., 67 n. 85; U. Betti, Diario del Concilio, 81; R. Burigana, La Bibbia al Concilio, op.cit., 433.

${ }^{88}$ Cf. DV 19: «La santa madre Chiesa ha ritenuto e ritiene con fermezza e con la più grande costanza che i quattro suindicati Vangeli, di cui afferma senza esitazione la storicità (quor rum historicitatem incunctanter affirmat), trasmettono fedelmente (fideliter tradere) quanto Gesù Figlio di Dio, durante la sua vita tra gli uomini, effettivamente operò e insegnò per la loro eterna salvezza, fino al giorno in cui fu assunto in cielo (cfr Ac 1,1-2). Gli apostoli poi, dopo l'Ascensione del Signore, trasmisero ai loro ascoltatori ciò che egli aveva detto e fatto, con quella più completa intelligenza delle cose, di cui essi, ammaestrati dagli eventi gloriosi di Cristo e illuminati dallo Spirito di verità, godevano».
} 
le internazionale" ${ }^{89}$ Secondo lo stesso Betti, ne fanno parte M. Lefèbvre, De Proença Sigaud e Carli. Betti parla anche di «posizione da consorteria». ${ }^{90}$

Furono ancora una volta Florit ${ }^{91}$ e van Dodewaard ad illustrare all'assemblea conciliare i risultati del lavoro svolto.

I risultati delle votazioni sono trascritti da P. Betti, che quanto ai voti nulli in realtà del tutto irrilevanti -, sarcasticamente annota: ${ }^{92}$ «il solito voto nullo dovuto ad un Padre intellettualmente assente o costituzionalmente retrivo». $\mathrm{O}$ ancora: «fanno pendant, per inutilità con i non placet». Lo stesso segnala un testimone diretto che ha fotografato «la mano nervosa di Mons. Carli che tracciava il suo ennesimo non placet»: ${ }^{93}$

\begin{tabular}{|c|c|c|c|c|c|}
\hline & & Votanti & Placet & Non placet & nulli \\
\hline & Voto preliminare & 2350 & 2345 & $6^{94}$ & \\
\hline \multirow{6}{*}{ 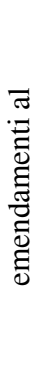 } & proemio e cap. I & 2194 & 2169 & 23 & 2 \\
\hline & Cap II & 2185 & 2123 & 55 & 7 \\
\hline & Cap III & 2189 & 2154 & 31 & 4 \\
\hline & Cap IV & 2188 & 2178 & 8 & 2 \\
\hline & Cap V & 2139 & 2115 & 19 & 5 \\
\hline & Cap VI & 2146 & 2126 & 14 & 6 \\
\hline & Voto sull'intero schema & 2115 & 2081 & 27 & 7 \\
\hline
\end{tabular}

\section{4 novembre 1965: Firenze - S. Maria del Fiore}

Alla presenza di quasi 500 padri, Florit celebra il $7^{\circ}$ centenario della nascita di Dante.

\section{8 novembre 1965}

Solo tre giorni prima della data decisiva del 18 novembre 1965, esattamente il 15 , il segretario generale notificò che la qualificazione teologica della Costi-

${ }^{89}$ Cf. U. Betti, Costituzione, op.cit., 64.

${ }^{90}$ Cf. U. Betti, Diario del Concilio, op.cit., 54; U. Betti, La rivelazione divina, 176; cf. anche R. Burigana, La Bibbia al Concilio, op.cit., 367.

${ }^{91}$ Cf. U. Betti, Costituzione, op.cit., 64 n. 80.

${ }^{92}$ U. Betti, Diario del Concilio, op.cit., 60.

${ }^{93}$ U. Betti, Diario del Concilio, op.cit., 69; id., U. Betti, Costituzione, op.cit., 65 n. 81; cf. anche R. Burigana, La Bibbia al Concilio, op.cit., 430-434.

${ }^{94}$ U. Betti, Diario del Concilio, op.cit., 80. 
tuzione sulla Divina Rivelazione era la stessa della Lumen Gentium (promulgata il 21 novembre 1964). Anche se il Concilio intende definire solo ciò che apertamente manifesta di voler definire, questa norma non si applica nel caso delle due costituzioni citate. Al tempo stesso tutto ciò che «ivi è insegnato, pur non sancito con una definizione dogmatica, è comunque dottrina del supremo magistero ecclesiastico, che quindi dev'essere accettata e ritenuta da tutti e singoli i fedeli». ${ }^{95}$

L'esito della votazione fu il seguente:

\begin{tabular}{|c|c|c|c|}
\hline Votanti & Placet & Non placet & nulli \\
\hline 2350 & 2344 & 6 & - \\
\hline
\end{tabular}

\section{Per una conclusione}

Non è semplice ricavare da tutte queste fonti - e forse neanche necessario una pur sommaria conclusione sul ruolo dell'arcivescovo, poi cardinale, Ermenegildo Florit. E tuttavia, rubando solo per un momento il mestiere allo storico, come del resto in queste note, appare provvidenziale l'origine e la scuola teologica romana di questo "fiorentino" di adozione, forse mai integrato nella realtà di questa terra. Fu solo la sua tenacia, sostenuta da P. Umberto Betti - poi card. Betti nella sua vecchiaia -, a dare ai Padri del Concilio la certezza che l'operazione che veniva conclusa era in linea con la tradizione, e tuttavia segnava un punto di non ritorno sulla Parola di Dio nella Chiesa, condotta attraverso la riflessione sulla Rivelazione divina, accanto al magistero e nel perenne dialogo fra Tradizione e Scrittura. Fu allora che i Padri dell'aula vaticana coniarono la splendida definizione della fede: l'obbedienza della fede al Dio che si rivela. ${ }^{96} \mathrm{Ma}$ c'era sempre un punto, di quella rivelazione che vede il Dio invisibile introdurre gli uomini, chiamati suoi amici, alla comunione con sé: ${ }^{97}$ cioè - e qui Florit si

${ }^{95}$ U. Betti, Costituzione, op.cit., 66 nn. 82 e 83.

${ }^{96}$ Cf. DV 5: «A Dio che rivela è dovuta "l'obbedienza della fede» (oboeditio fidei: Rm 16,26 , cfr. Rm 1,5, 2Co 10,5-6), con la quale l'uomo gli si abbandona tutt'intero (homo se totum libere Deo committit) e liberamente prestandogli "il pieno ossequio dell'intelletto e della volontà" e assentendo volontariamente alla Rivelazione che egli fa (plenum revelanti Deo intellectus et voluntatis obsequium praestando et voluntarie revelationi ab Eo datae assentiendo). Perché si possa prestare questa fede, sono necessari la grazia di Dio che previene e soccorre e gli aiuti interiori dello Spirito Santo, il quale muova il cuore e lo rivolga a Dio, apra gli occhi dello spirito e dia "a tutti dolcezza nel consentire e nel credere alla verità". Affinché poi l'intelligenza della Rivelazione diventi sempre più profonda, lo stesso Spirito Santo perfeziona continuamente la fede per mezzo dei suoi doni».

${ }^{97}$ Cf. DV 2: «Con questa Rivelazione infatti Dio invisibile (cfr. Col 1,15, 1Tm 1,17) nel suo grande amore parla agli uomini come ad amici (cfr. Ex 33,11, Jn 15,14-15) e si intrattiene con essi (cfr. Ba 3,38), per invitarli e ammetterli alla comunione con sé. Questa economia della Rivelazione comprende eventi e parole intimamente connessi, in modo che le opere, compiute da Dio nella sto- 
mostrò indubbiamente più titubante - in cui il Concilio apriva le Sacre Scritture all'intero popolo di Dio, perché permei l'intera vita della Chiesa. ${ }^{98}$ Proprio questo segna un punto di non ritorno, dopo tante stagioni di singolare distacco da quella fonte della spiritualità cristiana, o dalla stessa vita di fede, per non parlare della teologia, ${ }^{99}$ memori dell'affermazione di Girolamo che «l'ignoranza delle Scritture è ignoranza di Cristo». ${ }^{100}$

\section{Ermenegildo Florit and Dei Verbum}

\section{Summary}

The article by Stefano Tarocchi titled Ermenegildo Florit and "Dei Verbum" presents the contribution of the then Archbishop of Florence and his accompanying theologian Rev. Umberto Betti in the writing of the constitution on the Revelation of the Second Vatican Council. Based on the Diario del Concilio of the Florentine theologian and his correspondence with the Archbishop the author shows in a chronological order not only the participation of the Archbishop in the work of the theological commission that prepared subsequent versions of the document, but also scrupulously recounts the particular propositions modifying the versions of the document, expounding in this way the contribution of the two Florentines to the emergence of the constitution Dei Verbum against the background of subsequent stages of the shaping of this conciliar document.

\section{Keywords}

II Vatican Council, Constitution Dei Verbum, Ermenegildo Florit, Umberto Betti, Revelation

\section{Stowa kluczowe}

Sobór Watykański II, konstytucja Dei Verbum, Ermenegildo Florit, Umberto Betti, objawienie

ria della salvezza, manifestano e rafforzano la dottrina e le realtà significate dalle parole, mentre le parole proclamano le opere e illustrano il mistero in esse contenuto».

${ }^{98}$ DV 25: «Si accostino essi volentieri al sacro testo, sia per mezzo della sacra liturgia, che è impregnata di parole divine, sia mediante la pia lettura, sia per mezzo delle iniziative adatte a tale scopo e di altri sussidi, che con l'approvazione e a cura dei pastori della Chiesa, lodevolmente oggi si diffondono ovunque. Si ricordino però che la lettura della sacra Scrittura dev'essere accompagnata dalla preghiera, affinché si stabilisca il dialogo tra Dio e l'uomo; poiché «quando preghiamo, parliamo con lui; lui ascoltiamo, quando leggiamo gli oracoli divini».

${ }^{99}$ Cf. DV 24: «La sacra teologia si basa come su un fondamento perenne sulla parola di Dio scritta, inseparabile dalla sacra Tradizione; in essa vigorosamente si consolida e si ringiovanisce sempre, scrutando alla luce della fede ogni verità racchiusa nel mistero di Cristo. Le sacre Scritture contengono la parola di Dio e, perché ispirate, sono veramente parola di Dio, sia dunque lo studio delle sacre pagine come l'anima della sacra teologia. Anche il ministero della parola, cioè la predicazione pastorale, la catechesi e ogni tipo di istruzione cristiana, nella quale l'omelia liturgica deve avere un posto privilegiato, trova in questa stessa parola della Scrittura un sano nutrimento e un santo vigore».

${ }^{100}$ S. Girolamo, Comm. in Is., Prol. (PL 24, 17). 